\title{
Effects of oriented substrates on cell morphology, the cell cycle, and the cytoskeleton in Ros 17/2.8 cells
}

\author{
LI Hong, CHEN Juan, ZHANG Yan, SUN ShuJin, TAO ZuLai \& LONG Mian* \\ Key Laboratory of Microgravity, Institute of Mechanics, Chinese Academy of Sciences, and National Microgravity Laboratory and \\ Center for Biomechanics and Bioengineering, Institute of Mechanics, Chinese Academy of Sciences, Beijing 100190, China
}

Received May 11, 2009; accepted December 12, 2009

\begin{abstract}
Absence of gravity or microgravity influences the cellular functions of bone forming osteoblasts. The underlying mechanism, however, of cellular sensing and responding to the gravity vector is poorly understood. This work quantified the impact of vector-directional gravity on the biological responses of Ros 17/2.8 cells grown on upward-, downward- or edge-on-oriented substrates. Cell morphology and nuclear translocation, cell proliferation and the cell cycle, and cytoskeletal reorganization were found to vary significantly in the three orientations. All of the responses were duration-dependent. These results provide a new insight into understanding how osteoblasts respond to static vector-directional gravity.
\end{abstract}

substrate orientation, osteoblasts, cell shape, cell cycle, cytoskeleton

Citation: $\quad$ Li H, Chen J, Zhang Y, et al. Effects of oriented substrates on cell morphology, the cell cycle, and the cytoskeleton in Ros 17/2.8 cells. Sci China Life Sci, 2010, 53: 1085-1091, doi: 10.1007/s11427-010-4057-6

Bone loss is a fundamental risk in gravitational physiology for extended space flight. Human bone loss during spaceflight is up to $2 \%$ bone mass per month with exposure to a space microgravity environment [1,2]. Bone-forming osteoblasts are vital factors in mechanosensing and mechanotransduction in vivo, and the biological responses of osteoblasts in altered gravity provide basic understanding of human bone loss in space. Osteoblasts in vivo and in vitro were found to be affected by low gravity [3-6]. The underlying mechanism of osteoblastic gravisensing and gravitransduction, however, remains poorly understood.

Due to the limited opportunities for spaceflights and payload experiments, several simulated microgravity approaches on the ground have been proposed [7-9]. One of them is to employ a vector-averaged gravity approach (e.g., clinostat or random positioning machine), in which vector-directional gravity varies periodically and growing cells experience an averaged zero-like gravity, thereby leading to

*Corresponding author (email: mlong@imech.ac.cn) the so-called simulated microgravity on ground. Even though the bulk of experimental evidence has been obtained from such approaches, it remains unknown whether or not the observed responses are induced by simulated averaged zero-like gravity or by additional side effects. Vectoraveraged gravity is inevitably coupled with such extra forces as flow shear or hypergravity in a cell bioreactor, which are far greater than cell gravity itself $(\sim 1 \mathrm{pN})$. Thus, it is essential to quantify the impact of vector-directional gravity itself on cell responses.

For this purpose, a statically-oriented culture approach is according to the strategy that vector-directional gravity varies in a constant angle, rather than changing periodically in clinostat, with respect to a culture substrate. Anchorageindependent cells are highly asymmetric with regard to the direction of gravity in terms of cell shape and cellular structures. The viability of osteoblasts was diminished on the first day after being subjected to inverted cultures in downward-oriented substrates in static clinostat [10], where the constant angle between the gravity vector and the sub- 
strate vector is $180^{\circ}$. The dividing orientation of Chinese hamster ovary cell was randomly distributed with respect to gravity in a vertically-positioned culture flask [11], where the angle was $90^{\circ}$. Here we hypothesize that the cells responded to vector-directional gravity applied to the cells either through averaged-zero gravity or through a constant angle.

In this study, Ros 17/2.8 cells, well-known to be sensitive to mechanical stimuli and microgravity [12], were grown in an upward-, downward- or edge-on orientation, and cell morphology and nucleus translocation, cell proliferation and cell cycle, and cytoskeletal reorganization were quantified in a duration-dependent manner. Our results provide a new insight into understanding how osteoblasts respond to static vector-directional gravity.

\section{Materials and methods}

\subsection{Cells and reagents}

Rat osteosarcoma Ros 17/2.8 cells with a mature osteoblastic phenotype were purchased from the Peking University Health Science Center. The cells were grown at $37^{\circ} \mathrm{C}$ in DMEM (Hyclone) supplemented with $10 \%$ fetal calf serum (Hyclone), $2 \mathrm{mmol} \mathrm{L}^{-1}$ L-glutamine (Hyclone), and $1 \%$ penicillin-streptomycin (Sigma). FITC-conjugated phalloidin, mouse-anti-human vimentin monoclonal antibody, and FITC-conjugated goat-anti-mouse IgG were purchased from Sigma (USA).

\subsection{Cell proliferation on oriented substrates}

Glass coverslips (Corning, USA) were fabricated into a size of (11.0 \pm 0.1$) \mathrm{mm}$ wide and $(40.0 \pm 0.1) \mathrm{mm}$ long, and rinsed in the piranha solution and sterilized by autoclaves. Cells were grown on the coverslip for $8 \mathrm{~h}$ before being transferred onto a customer-made coverslip holder (made of polytetrafluoroethylene) respectively in the upward-, downward- or edge-on orientation (Figure 1). The assembly device was then put into a new Petri dish to grow for 5 days. A seeding density of $1 \times 10^{4}$ cells $\mathrm{cm}^{-2}$ was used for cell proliferation and cell cycle measurements. A relatively low density of 850 cells $\mathrm{cm}^{-2}$ was employed for cell morphology, nuclear translocation, and cytoskeletal reorganization measurements. To minimize the effect of hydrostatic pressure induced in the three orientations, upward- and downward-oriented coverslips were maintained at the same height with the same volume of culture medium of $40 \mathrm{~mL}$ per dish. Cells were grown with a culture medium of $60 \mathrm{~mL}$ per dish on the edge-on coverslip, in which culture cells were subjected to various hydrostatic pressures with a maximum difference of $11 \mathrm{~mm} \mathrm{H}_{2} \mathrm{O}$ height.

\subsection{Cell morphology and nuclear translocation}

Cultured cells were imaged using an inverted Zeiss fluorescence microscope (Axiovert 200, Germany) with a CCD camera (Hitachi HV D30, Japan) and a 20×phase contrast objective. Morphological analyses for $\sim 100$ individual cells without cell-cell contact were performed using Image J

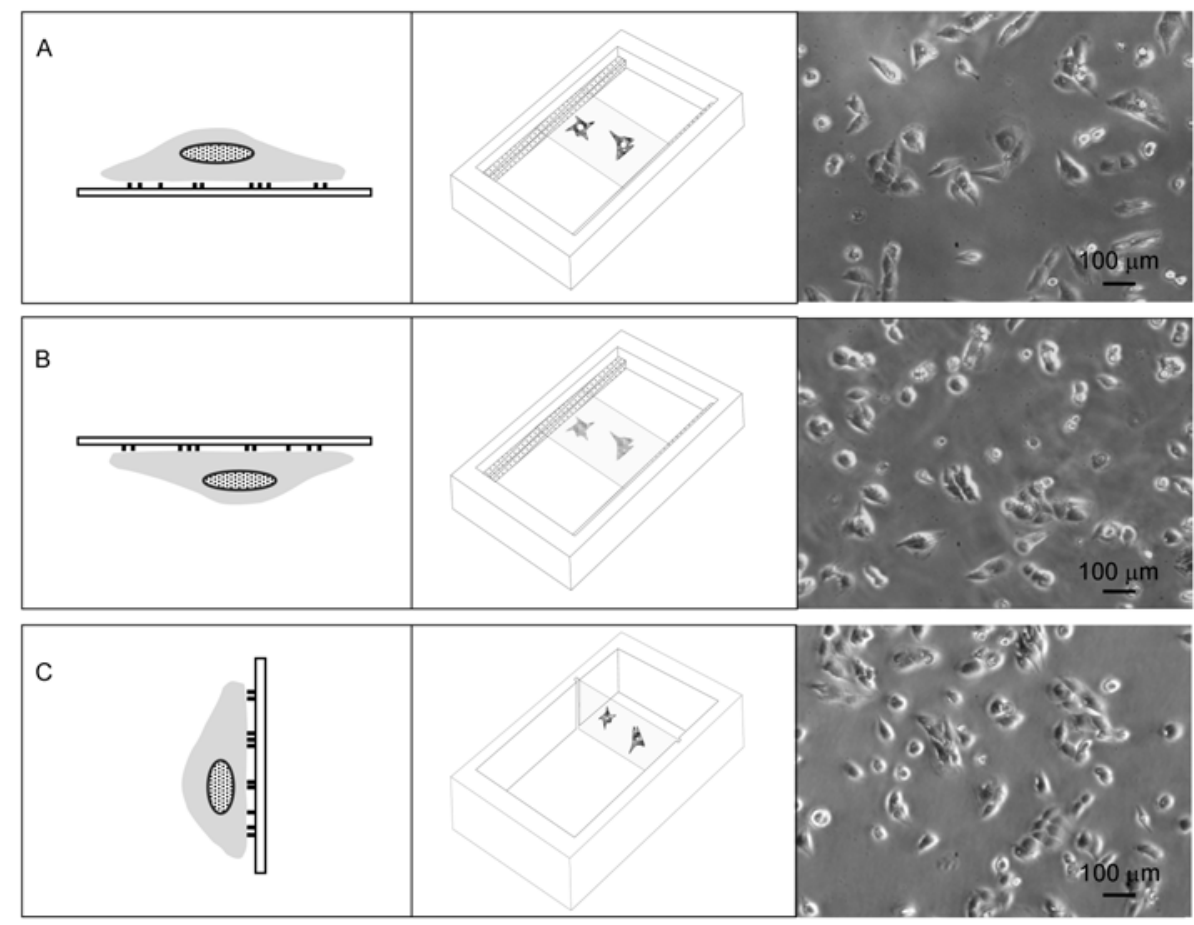

Figure 1 Ros 17/2.8 cells cultured on upward- (A), downward- (B) or edge-on- (C) oriented substrates. Schematic cell attachment (left column), culture system (middle column), and microscopic images of cultured cells (right column) were illustrated. Bars represent $200 \mu \mathrm{m}$. 
(NIH, USA) software. The projected cell area and cell shape factor $(S F)$ were measured, where $S F$, denoted as the perimeter $^{2} /(4 \times \pi \times$ area $)$, was used to quantify cell sphericity. The translocation distance, $D$, defined as the distance between two centroids of the projected cell contour and nucleus contour (Figure 2C), was gauged after flipping over the coverslip when the cells were grown in the downward or edge-on orientation. The measurements were conducted using three coverslips for each orientation in each run with three independent runs repeated. Data was then presented as the mean \pm standard error (SE). The statistical significance of (or the lack thereof) the difference was assessed by ANOVA.

\subsection{Cell cycle}

Cells were grown to confluence in a flask for two days to form contact inhibition which led to synchronization cells in the $\mathrm{G}_{0} / \mathrm{G}_{1}$ phase (about $85 \%-89 \%$ ) prior to being transferred onto a coverslip. After being cultured in the oriented substrate at given durations, cells were then trypsinized from the coverslip and the cell nuclei were stained using a $\mathrm{Cy}-$ cleTEST PLUS DNA Reagent Kit (Becton Dickinson). Cell cycle analysis was assessed using flow cytometry (FACSCalibur, Becton Dickinson, USA), and the fluorescence histograms were obtained using manufacturer-provided ModFit software. The measurements were performed at least in triplicate.

\subsection{Cytoskeletal reorganization}

The cells on the coverslips were rinsed in ice-cold PBS three times, fixed with $3.7 \%$ paraformaldehyde for $10 \mathrm{~min}$ at $4{ }^{\circ} \mathrm{C}$, and permeablized by $0.1 \%$ Triton $\mathrm{X}-100$ for $5 \mathrm{~min}$ at $4^{\circ} \mathrm{C}$. Cells were washed and incubated in $1 \% \mathrm{BSA} / \mathrm{PBS}$ for $30 \mathrm{~min}$ at $37^{\circ} \mathrm{C}$ to block non-specific staining. To visualize
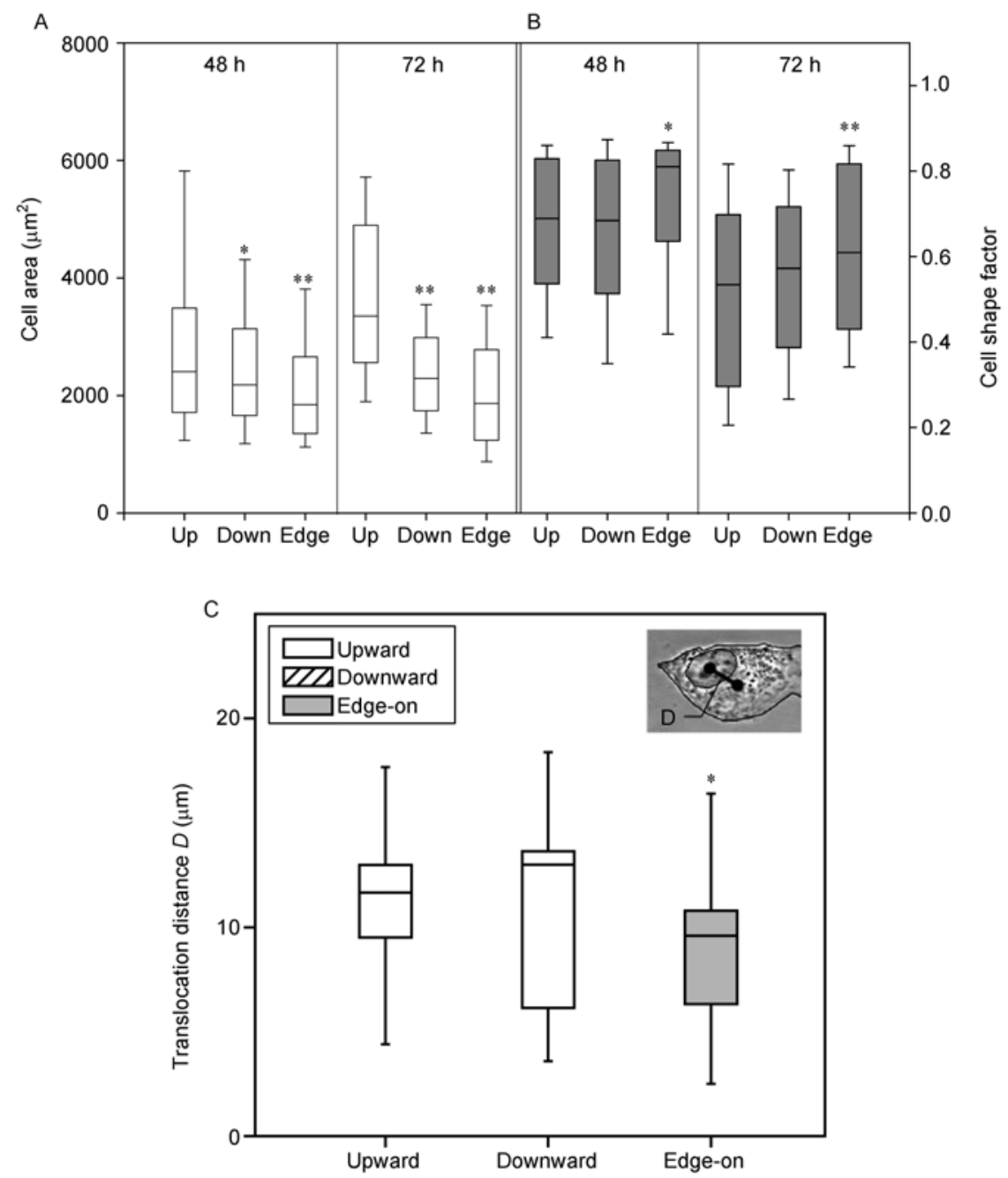

Figure 2 Cell area (A) and shape factor (B) at 48 and $72 \mathrm{~h}$ as well as nucleus translocation distance (C) at $72 \mathrm{~h}$, for Ros $17 / 2.8$ cells cultured in three orientations. Data is presented, using a box-and-whiskers plot, as the median (median line within box) with the 25th and 75th percentiles (upper and lower lines of box), and the 10th and 90th percentiles (whiskers above and below box). Significant differences: $*, P<0.05$ and $* *, P<0.01$ as compared to that for upward-cultured cells. 
filamentous actin, cell preparations were incubated by FITC-conjugated phalloidin for $60 \mathrm{~min}$ at $37^{\circ} \mathrm{C}$, and fully washed in PBS and fixed in $50 \%$ glycol/PBS. To visualize intermediate filament vimentin, cells were fixed, permeablized, and blocked with BSA as described above. After being incubated with the mouse-anti-human vimentin monoclonal antibody for $60 \mathrm{~min}$, cells were rinsed three times in PBS and incubated with FITC-conjugated goat-anti-mouse IgG at a dilution of $1: 100$ for $60 \mathrm{~min}$ at $37^{\circ} \mathrm{C}$. Actin and vimentin distributions were observed using a laser scanning confocal microscope (Leica TCS-SP II, Germany) with a $63 \times$ oil-immersion objective at respective excitation and emission wavelengths of 490 and $550 \mathrm{~nm}$. Images were acquired at a speed of 400 lines $\mathrm{s}^{-1}$, and eight images were averaged to enhance the signal-to-noise ratio. F-actin polymerization and the vimentin network were quantified by calculating the averaged relative fluorescence intensity (RFU) of an ensemble of cells after background subtraction using ANOVA.

\section{Results and discussion}

\subsection{Responses of cell morphology remodeling and cell nucleus translocation}

To maximize the effect of substrate orientation on cell morphological remodeling, Ros 17/2.8 cells were respectively grown for $>40 \mathrm{~h}$ on the upward- (Figure 1A), downward- (Figure 1B) or edge-on- (Figure 1C) oriented substrate. It was found that the cell area in the downward orientation was reduced at 48 and 72 h, i.e., 1.6- and 1.8-fold lower in the downward and edge-on orientations (respectively $(2429.1 \pm 917.1)$ and $\left.(2106.2 \pm 1166.8) \mu^{2}\right)$ compared with that in the upward orientation at $72 \mathrm{~h}((3762.4 \pm 1707.1)$ $\mu \mathrm{m}^{2}$ ) (Figure 2A; $P<0.01$ ). Calculated cell shape factors in the edge-on orientation $((0.72 \pm 0.18)$ and $(0.61 \pm 0.20))$ were higher than those in the downward orientation $((0.65 \pm 0.19)$ and $(0.55 \pm 0.19))$ and in the upward orientation $((0.66 \pm 0.17)$ and $(0.51 \pm 0.22))$ at 48 and $72 \mathrm{~h}$ (Figure $2 \mathrm{~B} ; P<0.01$ or 0.05 ). This data suggested that cells in the downward orientation retained sphericity while those in the edge-on orientation were more likely to become round and significantly shrink.

In the downward and edge-on substrate orientations, high-density nuclear structures attempted to relocate towards the apical surface and lateral membrane of cells $[13,14]$, which in turn applied extra pressure on the cytoskeletal fibers adjacent to the nucleus. Thus we also tested to determine if the cell nucleus is able to differently translocate when cells grow on oriented substrates. The translocation distance, $D$, between two centroids of the projected cell contour and nucleus contour in the $x-y$ plane was measured, where the normal vector of the $x-y$ plane is parallel to the gravitational vector in the upward and downward orientations (respectively $180^{\circ}$ and $0^{\circ}$ ), and perpendicular to the gravitational vector in the edge-on orientation $\left(90^{\circ}\right)$. As exemplified in Figure $2 \mathrm{C}$, the mean translocation distance in the edge-on orientation $((9.50 \pm 3.51) \mu \mathrm{m})$ was 1.2 and 1.2 -fold lower $(P<0.05)$ but no differences were found between the upward and downward orientations (respectively $(11.61 \pm 2.84)$ and $(11.44 \pm 4.3) \mu \mathrm{m})$. These results indicated that the nucleus translocation along with the gravitational vector was significantly restrained in the edge-on orientation, suggesting that the biological responses of the osteoblasts to vector-directional gravity are correlated with the dynamic translocation of the cell nucleus.

Cell shape and sphericity were regulated during spaceflight and in statically vector-directional or dynamically vector-averaged gravity. Ros 17/2.8 cells subjected to spaceflight and parabolic flight exhibited reduced cell areas with higher irregularity of cell shape [15-17]. MC3T3-E1 cells appeared to be more round-shaped in spaceflight [18]. Mechanical deformation of the upward-oriented substrate induced the decrease of cell area following a bound-up to the baseline level in Ros 17/2.8 cells [17]. Similar responses were found in the current work where the cell area in the downward and edge-on orientations initially reduced within the first 3-day (Figure 2A) before returning to the same level as that in the upward orientation, suggesting that the cell morphological remodeling to vector-directional gravity is duration-dependent. This remodeling was further observed from a slight lag-phase of cell growth in the downward and edge-on orientations at 24 or $48 \mathrm{~h}$ (Figure $3 \mathrm{~A}$ be-

A
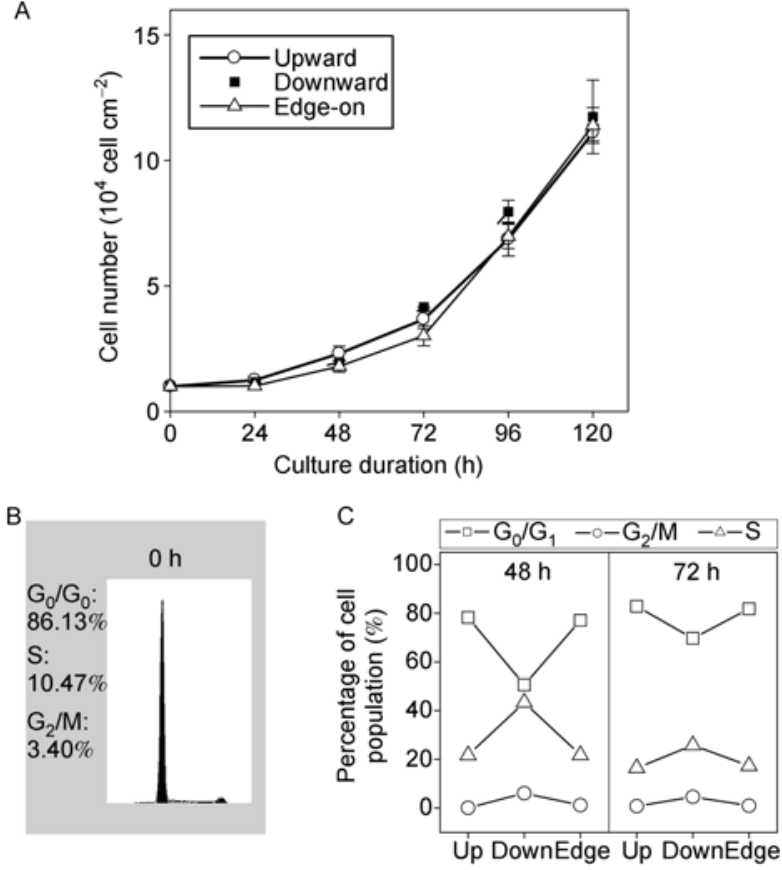

Figure 3 (A) Time course of Ros 17/2.8 cell proliferation in three orientations. Data is presented as the mean \pm standard error (SE) of three independent measurements. (B, C) Cell cycle analysis for Ros 17/2.8 cells in three orientations at 0 (B), 48 and 72 (C) h. Cells were synchronized in the $\mathrm{G}_{0} / \mathrm{G}_{1}$ phase prior to being transferred to an oriented substrate. 
low), which was consistent with those observations under microgravity and in clinostat $[18-21,26]$.

\subsection{Responses of cell proliferation and the cell cycle phase}

The time course of cell growth was independently measured for different oriented substrates. As exemplified in Figure $3 \mathrm{~A}$, cell growth exhibited a lag-phase within first two days, followed by a logarithm-phase for up to five days. No statistically-significant differences in cell proliferation were found in the three orientations although cell growth in the first three days was slightly reduced in the downward and edge-on orientations. These results indicated that vector-directional gravity had no impact on cell growth in the oriented substrates.

We further quantified the effect of oriented substrates on cell division. Here cell cycle analysis was performed at different culture durations. Ros 17/2.8 cells were pre-cultured in a flask over $85 \%$ confluence to synchronize the cells in the $\mathrm{G}_{0} / \mathrm{G}_{1}$ phase, as seen in Figure $3 \mathrm{~B}$, prior to being trypsinized and transferred onto an oriented substrate. Flow cytometry analyses indicated that the percentage of $S$-phase cells was significantly enhanced and the percentage of $\mathrm{G}_{0} / \mathrm{G}_{1}$-phase cells was sharply reduced when Ros $17 / 2.8$ cells were grown in the downward orientation for 48 and 72 $\mathrm{h}$ but no differences were found in the edge-on orientation, as compared to those in the upward orientation (Figure 3C). In the downward orientation, the $S$-phase cell percentage had a significant decrease and $\mathrm{G}_{0} / \mathrm{G}_{1}$-phase cell percentage increased with culture durations, while no duration dependence of cell cycle phases was found in the edge-on orientation (Figure 3C). Taken together, these results indicated that cell division, rather than cell proliferation, was regulated in the downward orientation but not in the edge-on orientation.

Cell division is regulated by microgravity or vector-directional gravity. Spaceflight induced a prolonged mitosis of MCF-7 cells and less focal adhesion sites in the post-mitosis of Ros 17/2.8 cells [19-20]. Dynamically vector-averaged gravity in clinostat enhanced the numbers of $S$-phase osteoblasts [10] but no significant difference in the $\mathrm{G}_{2} / \mathrm{M}$-phase percentage was observed when Ros 17/2.8 cells were cultured under microgravity and on the ground [20]. In the current study, an enhancement in $\mathrm{S}_{-} \mathrm{G}_{2} / \mathrm{M}$-phase percentage was found when Ros 17/2.8 cells were grown up in the downward orientation (Figure 3C). Cell cycle responses depend on how cells are synchronized prior to being transferred to an oriented substrate. The percentages in the specific phases of the upward and edge-on orientations were found to be different when a low synchronization percentage (65.6\%) was applied to Ros 17/2.8 cells (data not shown).

\subsection{Responses of cytoskeletal reorganization}

To understand the impacts of oriented substrates on cy- toskeletal reorganization, microfilament and intermediate filament distributions were observed in oriented substrates. As exemplified in Figure 4, cytoskeletal actin stress fibers formed a uniform network within a Ros 17/2.8 cell grown in an upward orientation (Figure 4A). More abundant actin stress fibers were found when the cells were grown in the downward and edge-on orientations (Figures $4 \mathrm{~B}$ and $4 \mathrm{C}$ ), implying that the actin network was strengthened by adapting vector-directional gravity. It was also observed that actin stress fibers were bundled up or formed tight clusters at the cell periphery (arrows in Figures 4B and 4C). By contrast, vimentin filaments were diffusely distributed in the cytoplasm and formed a focal filamentous network when cells were grown in an upward orientation (Figure 4D). Particularly, some filaments in the vicinity of the nucleus formed a condensed cord-like center, from which other filaments radiated outward to cell periphery (arrows in Figure 4D). More abundant filaments were observed in the cytoplasm, and the smaller, rounder cord-like center was formed in the vicinity of the nucleus when cells were grown in a downward orientation (arrows in Figure 4E). It was evident that vimentin was uniformly distributed throughout the cytoplasm with a smaller, rounder cord-like center in the edge-on orientation (arrows in Figure 4F).

Cytoskeletal reorganization was further quantified using RFU. The fluorescent intensity of actin or vimentin filaments was measured for a total of 20 cells in each orientation and the average content of protein of interest per cell was presented in Figure 4G-H. It was indicated that the RFU of F-actin was 1.3-fold higher in the downward and edge-on orientations (respectively $(26.7 \pm 5.5)$ and $(26.7 \pm$ 7.3)) than in the upward orientation (20.5 \pm 5.0$)$ (Figure 4G; $P<0.01)$. The RFUs of vimentin were not significantly dif-

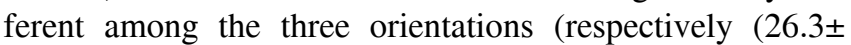
5.7), (23.6 \pm 3.1$)$, and $(25.0 \pm 6.3)$ ) (Figure $4 \mathrm{H} ; P>0.1$ ), imparting the confidence of the above observations. Taken together, these results provided sub-cellular evidence to support the concept that intracellular cytoskeletal reorganization was required for cellular responses to oriented substrates.

The cytoskeletal network is crucial for maintaining cell integrity and to respond to external mechanical stimuli. In conventional cell attachment in the upward orientation, the denser nucleus tends to settle downward to the bottom and the interconnected cytoskeleton maintains the nucleus in place. In a downward or edge-on orientation, however, high-density organelles relocate towards the apical surface or the lateral membrane and pull down the cytoskeletal network inside the cell. Less-organized actin stress fibers were found for Ros 17/2.8 cells in spaceflight and parabolic flight [15-17]. The loosening of perinuclear cytokeratin was observed when MCF-7 cells were subjected to $48 \mathrm{~h}$ of weightless space flight [19], implying that the perinuclear network might be changed for different cell attachment orientations. In the current study, cytoskeletal reorganization 

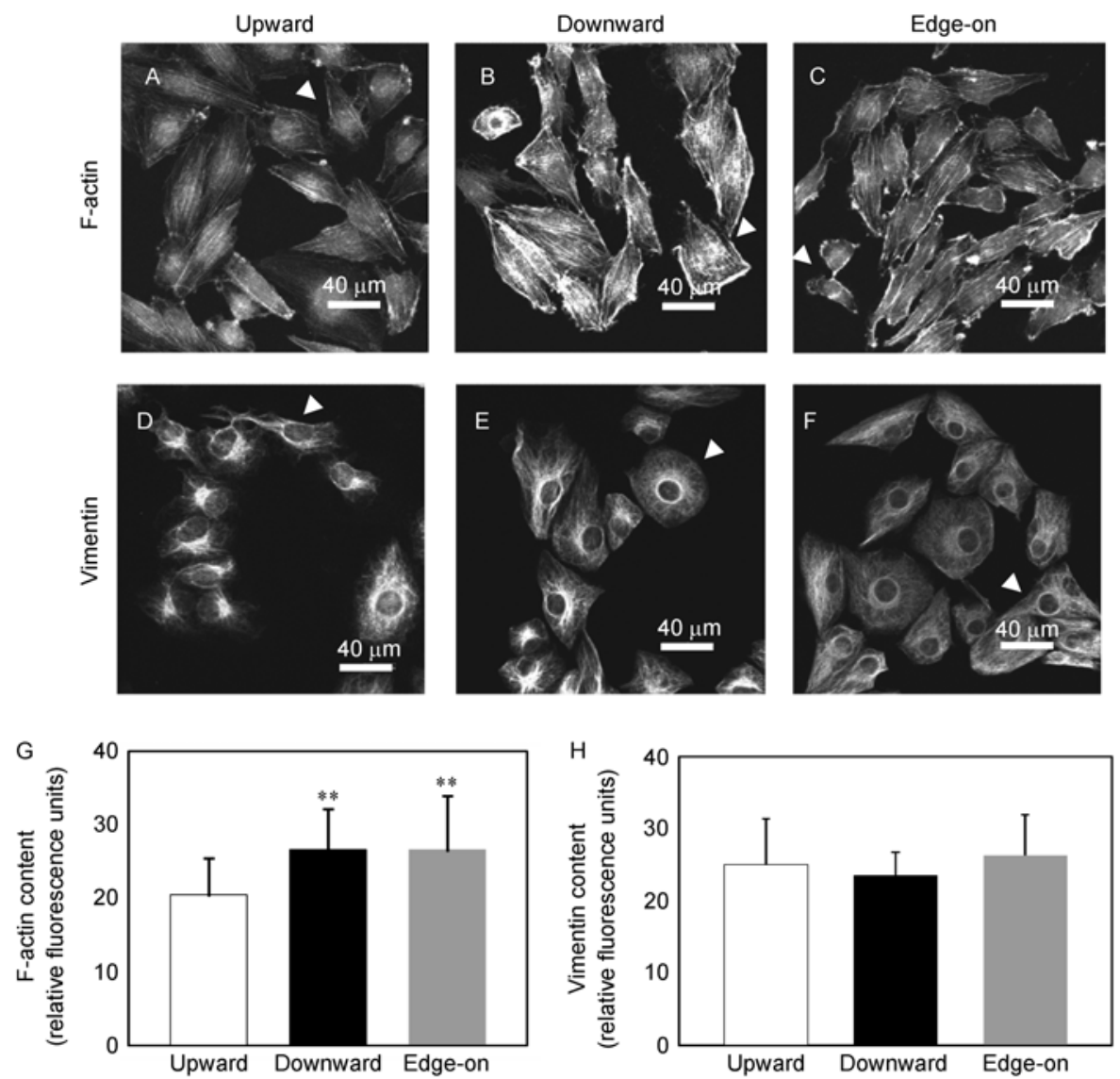

Figure 4 (A-F) Typical cytoskeleton reorganization of the actin (A-C) and vimentin (D-F) network for Ros 17/2.8 cells cultured in an upward (A, D), downward (B, E) or edge-on (C, F) orientation at $120 \mathrm{~h}$. Arrows indicate a well-organized vimentin network and bars represent $40 \mu \mathrm{m}$. (G, H) Relative fluorescent intensities of F-actin $(\mathrm{G})$ or vimentin $(\mathrm{H})$ content for Ros 17/2.8 cells in the upward (open bars), downward (black bars) or edge-on (grey bars) orientation at 120

h. Data is presented as the mean \pm SE. Significant difference: $* *, P<0.01$ as compared to that for upward-cultured cells.

of the actin and vimentin network was also observed when Ros $17 / 2.8$ cells were grown in the downward or edge-on orientation (Figure 4). Our data concerning the nucleus translocation distance (Figure 2B) also indicated that the tendency that high-density nucleus settled along the gravity vector was confined, presumably due to the enhanced actin polymerization and the reorganized perinuclear vimentin network (Figure 4). Although further analyses are required for three-dimensional cell morphology and translocation, these results suggested that the translocation of a high-density nucleus and other organelles is regulated by cytoskeletal reorganization in vector-directional gravity.

\section{Conclusion}

We used a substrate-oriented approach to quantify the responses of osteoblasts to static vector-directional gravity, in terms of "normal" (upward), "inverted" (downward), and "erected" (edge-on) static culture systems. Our data concerning cellular responses increase the understanding of the cellular gravisensing mechanism by combining with dy- namically vector-averaged gravity measurements as well as from payload experiments under microgravity.

The authors wish to thank Dr. Dong Hongbin for providing technical support. This work was supported by the Knowledge Innovation Program of the Chinese Academy of Sciences (Grant No. KJCX2-YW-LO8), the National Natural Science Foundation of China (Grant Nos. 30730032 and 30730093), the National Key Basic Research Foundation of China (Grant No. 2006CB910303), and the National High Technology Research and Development Program of China (Grant No. 2007AA02Z306).

1 Lang T F, Evans H J, Lu Y. Adaptation of the proximal femur to skeletal reloading after long-duration spaceflight. J Bone Miner Res, 2006, 21: 1224-1230

2 Sibonga J D, Sung H G, Spector E R, et al. Recovery of spaceflight-induced bone loss: bone mineral density after long-duration missions as fitted with an exponential function. Bone, 2007, 41: 973-978

3 Vico L, Lafage-Proust M H, Alexandre C. Effects of gravitational changes on the bone system in vitro and in vivo. Bone, 1998, 22: 79S- $82 \mathrm{~S}$

4 Hughes-Fulford M. Physiological effects of microgravity on osteoblast morphology and cell biology. Adv Space Biol Med, 2002, 8: $129-157$

5 Hughes-Fulford M, Jütting U. Reduction of anabolic signals and al- 
teration of osteoblast nuclear morphology in microgravity. J Cell Biochem, 2006, 99: 435-449

6 Bucaro, M A, Risbud M V, Ayyaswamy P S, et al. The effect of simulated microgravity on osteoblasts is independent of the induction of Apoptosis. J Cell Biochem, 2007, 102: 483-495

7 Klaus D M. Clinostats and bioreactors. Gravit Space Biol Bull, 2001, 14: 55-64

8 Schwarzenberg M, Pippia P, Meloni M A, et al. Signal transduction in T lymphocytes--a comparison of the data from space, the free fall machine and the random positioning machine. Adv Space Res, 1999, 24: 793-800

9 Patel M J, Sykes M C, Ward N E, et al. Identification of mechanosensitive genes in osteoblasts by comparative microarray studies using the rotating wall vessel and the random positioning machine. $\mathrm{J}$ Cell Biochem, 2007, 101: 587-599

10 Kacena M A, Todd P, Gerstenfeld L C, et al. Experiments with osteoblasts cultured under varying orientations with respect to the gravity vector. Cytotechnology, 2002, 39: 147-154

11 Helmstetter C E. Gravity and the orientation of cell division, Proc Natl Acad Sci USA, 1997, 94: 10195-10198

12 Guignandon A, Usson Y, Laroche N, et al. Effects of intermittent or continuous gravitational stresses on cell-matrix adhesion: quantitative analysis of focal contacts in osteoblastic ROS 17/2 8 cells. Exp
Cell Res, 1997, 236: 66-75

13 Worofka R, Sauermann G, Nuclear columns, kinetics of RNA synthesis and release in isolated rat liver nuclei. Biochim Biophys Acta, 1978, 518: 61-80

14 Wyrobek A J, Meistrich M L, Furrer R, et al. Physical characteristics of mouse sperm nuclei. Biophys J, 1976, 16: 811-825

15 Guignandon A, Vico L, Usson Y, et al. Shape changes of osteoblastic cells under gravitational variations during parobolic flight-Relationship with PGE2 synthesis. Cell Struct Funct, 1995, 20: 369-375

16 Guignandon A, Genty C, Vico L, et al. Demonstration of feasibility of automated osteoblastic line culture in space flight. Bone, 1997, 20: $109-116$

17 Guignandon A, Akhouayri O, Usson Y, et al. Focal contact clustering in osteoblastic cells under mechanical stresses: microgravity and cyclic deformation. Cell Commun Adhes, 2003, 10: 1-15

18 Hughes-Fulford M, Lewis M L. Effects of microgravity on osteoblast growth activation. Exp Cell Res, 1996, 224: 103-109

19 Vassy J, Portet S, Beil M, et al. Effect of weightlessness on cytoskeleton architecture and proliferation of human breast cancer cell line MCF-7. FASEB J, 2001, 15: 1104-1106

20 Guignandon A, Lafage-Proust M, Usson Y, et al. Cell cycling determines integrin-mediated adhesion in osteoblastic ROS 17/2 8 cells exposed to space-related conditions. FASEB J, 2001, 15: 2036-2038 